\title{
Semantic web service search: a brief survey
}

Klusch, Matthias ; Kapahnke, Patrick ; Schulte, Stefan ; Lecue, Freddy ; Bernstein, Abraham

\begin{abstract}
Scalable means for the search of relevant web services are essential for the development of intelligent service-based applications in the future Internet. Key idea of semantic web services is to enable such applications to perform a high-precision search and automated composition of services based on formal ontology-based representations of service semantics. In this paper, we briefly survey the state of the art of semantic web service search.
\end{abstract}

DOI: https://doi.org/10.1007/s13218-015-0415-7

Posted at the Zurich Open Repository and Archive, University of Zurich

ZORA URL: https://doi.org/10.5167/uzh-134977

Journal Article

Accepted Version

Originally published at:

Klusch, Matthias; Kapahnke, Patrick; Schulte, Stefan; Lecue, Freddy; Bernstein, Abraham (2016). Semantic web service search: a brief survey. Künstliche Intelligenz (KI), 30(2):139-147.

DOI: https://doi.org/10.1007/s13218-015-0415-7 


\title{
Semantic Web Service Search: A Brief Survey
}

\author{
Matthias Klusch • Patrick Kapahnke • Stefan Schulte • Freddy Lecue • \\ Abraham Bernstein
}

Received: date / Accepted: date

\begin{abstract}
Scalable means for the search of relevant web services are essential for the development of intelligent service-based applications in the future Internet. Key idea of semantic web services is to enable such applications to perform a high-precision search and automated composition of services based on formal ontology-based representations of service semantics. In this paper, we briefly survey the state of the art of semantic web service search.
\end{abstract}

Matthias Klusch, Patrick Kapahnke

German Research Center for Artificial Intelligence

Agents and Simulated Reality Department

Intelligent Information Systems Research Team

Stuhlsatzenhausweg 3, 66123 Saarbruecken, Germany

E-mail: \{matthias.klusch, patrick.kapahnke\}@dfki.de

Stefan Schulte

TU Wien

Distributed Systems Group

Argentinierstrasse 8/184-1, 1040 Wien, Austria

E-mail: s.schulte@infosys.tuwien.ac.at

Freddy Lecue

IBM Research

Smarter Cities Technology Center

Dublin, Ireland

E-mail: freddy.lecue@ie.ibm.com

Abraham Bernstein

Department of Informatics

University of Zurich

Binzmuehlestrasse 14, CH-8050 Zurich

E-mail: bernstein@ifi.uzh.ch

\section{Introduction}

At present, there are tens of thousands of web services for a huge variety of applications and in many heterogeneous formats available for the common user of the web. Scalable means for a high-precision search of relevant services with minimal human intervention are essential for the development of intelligent service-based applications in the Internet. In fact, there is a broad range of tools and systems for the search of web services described in XML-based WSDL (Web Service Description Language), WADL (Web Application Description Language) or REST APIs which do not provide any formal service semantics [24]. On the other hand, since the early 2000s, the semantic web community developed quite a few and sophisticated solutions for the search of semantic web services in different description languages and formats, and applications.

Key idea of semantic web services is to annotate web services with concepts which are defined in formal logic-based ontologies such that, from an AI perspective, intelligent agents and service-based applications can actually reason on such formal service semantics. In contrast to web service descriptions, this may facilitate not only the semantic interoperation between services but their automated logic-based composition planning and a more precise service search. In fact, it has been shown at an early stage of this research area that an appropriate combination of non-logic-based and logic-based semantic service selection can significantly 
outperform both kinds of selection in terms of precision but at the cost of higher response times [28].

Technologies for the search of semantic web services have been employed in different application domains such as semantic service-based business process management, smart factories [44], smart health care [56], virtual 3D web environments [22], and social media [32]. However, the practical trade-offs between the advantages of applying such technologies and the additional manual and computational efforts required to achieve them in different technical infrastructures and application settings still remain to be more deeply investigated within comparative studies.

In this survey, we provide a short overview of stateof-the-art approaches to the search of semantic web services in terms of their classification and brief descriptions of representative examples. The remainder of this paper is structured as follows. A short introduction to semantic service descriptions in Sect. 2 is followed by a brief survey of approaches to their search in Sect. 3 before we conclude in Sect. 4. For a survey on web service search, we refer to, for example, [24]. ${ }^{1}$

\section{Semantic Service Description}

Semantic web services (in short: semantic services) are web services whose functional and non-functional semantics are described in machine-understandable form with formal ontology-based annotations. On an abstract level, a semantic web service description consists of a semantic profile and a semantic process model, which describe the semantics of the service it is grounded in, in terms of what the service does and how it actually works, respectively (cf. Fig. 1) [26].

More concrete, the semantic profile describes the functional semantics of the service in terms of semantic annotations of its input (I) and output (O) parameters, as well as logic-based description of preconditions (P) and effects (E) which are supposed to hold before or after executing the service in a given world state. In addition, the profile includes semantically annotated non-functional service parameters which are concerned with service provenance and quality of service (QoS) covering, for example, delivery constraints, cost model with rules for pricing, repudiation, availability, and privacy policy. The semantic process model describes how

1 The paper contains revised parts from [24-27].

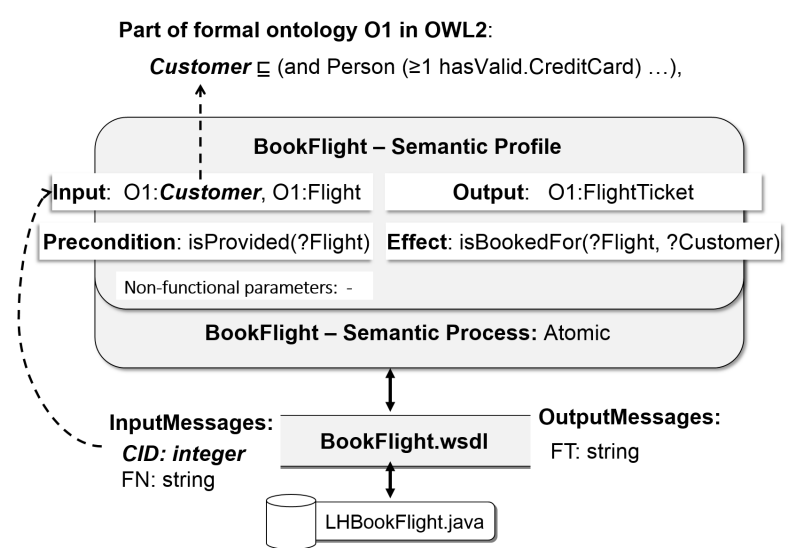

Fig. 1 Abstract simple example of a semantic web service grounded in a WSDL service

the service internally works in terms of the interplay between data and control flow for its subservice interactions with appropriate workflow operators and control constructs like sequence, split+join, and choice. According to the semantic service-oriented architecture (SOA) paradigm, semantic services are grounded in executable web services such as WSDL or RESTful services.

Prominent languages and formats for semantic service description are OWL-S (Web Ontology Language for Web Services) [45], WSML (Web Service Modeling Language) [13], the W3C standard SAWSDL (Semantic Annotations for WSDL and XML Schema) [12], USDL (Unified Service Description Language) [36,48], Linked USDL [49], as well as the microformats hRESTS [37], SA-REST [16], and MicroWSMO. These description models mainly differ in their formal logic-based foundation and the possible extent of service annotation $[26,50]$.

For example, in OWL-S the service input/output parameters $(\mathrm{I} / \mathrm{O})$ parameters are annotated with concepts which are exclusively defined in the formal logicbased W3C standard web ontology language OWL2 ${ }^{2}$. OWL-S service preconditions $(\mathrm{P})$ and effects $(\mathrm{E})$ can be specified, for example, in the formal semantic web rule language SWRL ${ }^{3}$ or in PDDL (Planning Domain Definition Language). SAWSDL allows to annotate WSDL service elements with references to web resources of any media type such as plain text, video, picture, audio podcast, and concepts defined in a formal logic-

\footnotetext{
2 OWL2: www.w3.org/TR/owl2-overview/

3 SWRL: www.w3.org/Submission/SWRL/
} 
based ontology. The same approach is taken in the SAREST framework for semantically annotating RESTful services. Both SAWSDL and SA-REST do not allow the specification of preconditions and effects, and the handling of semantic annotations is completely outside these languages. In this sense, unlike OWL-S and WSML, neither SAWSDL nor SA-REST provide services with a unique formal model of their semantics.

\section{Semantic Service Search}

Semantic service search can be performed in different ways depending on how the services of the considered search space are described, how the search process is organized, and which means of service selection are used for the search.

\subsection{Classification and Evaluation}

Current approaches for the search of semantic services can be classified as either centralized or decentralized directory-based, or as decentralized and directory-less.

Directory-based search. Service providers are supposed to register their services with either one central and possibly replicated directory, or multiple distributed (federated) service directories at distinguished nodes of the network, while service consumers are informed about available services in the network only through these directory nodes.

A centralized directory-based search of relevant semantic services can be performed by using either a contemporary web search engine, or a specialized semantic web service search engine, or a dedicated and authoritative semantic web service directory with a query interface. In any case, though in compliance with the general W3C web service interaction lifecycle for (semantic) SOA, such a central semantic service directory is acting as an intermediary between service provider and consumer but represents a potential single pointof-failure and performance bottleneck for service-based applications.

A decentralized directory-based search for semantic services can be performed in a structured P2P network overlay with a respective query routing protocol. Semantic services are placed at and discovered by all peer nodes according to a global service distribution or replication scheme, and the location mechanism of the network. In this regard, directory-based search corresponds to a structured search for services in multiple peer directories according to a given overlay such as in distributed hash table (DHT) based Chord rings, compound routing indices, or a hierarchic federation of service directories with super-peers. Semantic service search in structured P2P networks provides a search guarantee in the sense of total recall and logarithmic complexity in the size of the network for finding popular, i.e. highly replicated, as well as rare services. On the other hand, it comes at the cost of high communication overhead for publishing and maintaining the structured overlay when peers are joining or leaving the network, or if the set of services which they provide changes.

Directory-less search. Directory-less search for semantic services is performed in unstructured P2P networks without any given overlay structure. In this case, each peer initially knows only about services provided by its own or its direct neighbor peers. Prominent examples of location or query routing schemes in such networks are query flooding and k-random walks with replication and caching strategies, as well as informed probabilistic adaptive search. This type of $\mathrm{P} 2 \mathrm{P}$ search is known to be effective for finding popular but not rare items such as semantic services and provides only probabilistic search guarantees, i.e., incomplete recall. Finally, service discovery in hybrid P2P networks with structured and unstructured overlay parts can be performed by routing service requests to super-peers in the structured overlay part in order to find relevant rare services, or processed with restricted flooding or broadcasting to peers of the unstructured network part to find relevant popular services. One challenge is to let peers which are members of both types of network parts deliberatively decide on when to best and how to switch between the respective protocols during the search.

Semantic selection. Any approach to semantic service search depends, in particular, on the used method for semantic service selection, also known as semantic service matchmaking. The process of service selection or matchmaking encompasses (a) the pairwise semantic matching of a given service request with each service that is registered with the matchmaker in his local service directory, and (b) the semantic relevance ranking of these services. In contrast to service brokers, 
a service matchmaker only returns a rank list of relevant services and related provenance information to its human user or application but does not handle the interaction with selected services. Current approaches for semantic service matching can be classified as either logic-based semantic, non-logic-based semantic, or hybrid semantic depending on the nature of reasoning means that are used for this purpose. Non-logicbased semantic matching exploits, for example, means of graph matching, schema matching, data mining, and text similarity measurement, while logic-based semantic matching performs logical reasoning on service descriptions. Examples of the latter are the logical I/O concept subsumption-based plugin matching of service signatures, and the logical specification plugin matching of preconditions and effects.

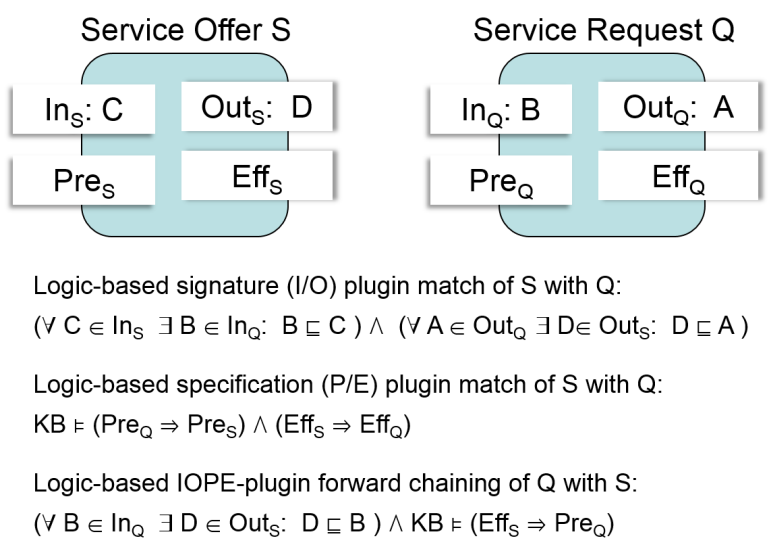

Fig. 2 Logic-based IOPE-plugin matching and chaining of semantic services.

Logical and full functional (IOPE) profile matching combines the scores of logical signature (IO) and specification (PE) matching. Hybrid semantic matching is a combination of both types of matching. Adaptive matchmakers are capable of learning how to best aggregate different matching filters off or on line. Representative examples of each of these types of semantic service matchmaking are provided later on. In any case, it is commonly assumed that service requests and offers are given to the matchmaker in the same format, or are appropriately transformed by the matchmaker itself. Besides, semantic service matchmakers can, in principle, be part of any kind of semantic service search through their use, for example, in the query interface of a central directory, or multiple federated directories, or local directories owned by peers in an unstructured P2P network.

Evaluation. Any system for semantic service search can be evaluated according to the following five criteria: (1) its support of different service description formats and languages, (2) its usability and required amount of effort for its configuration, (3) its support of service composition planning through, for example, context-aware pruning of the search space or interactive recommendations for a step-wise forward or backward chaining of services by the user, (4) its user data privacy policy, and (5) the service retrieval performance in terms of correctness and average query response time (AQRT) over given service test collections. The correctness of semantic service search is commonly evaluated with classical information retrieval measures such as average precision (AP) and macro-averaged precision at standard recall levels for binary relevance, as well as the normalized discounted cumulative gain or Q-measure for graded relevance. Current benchmarking initiatives for semantic service search and composition include the international SWS challenge for semantic web service composition, the WS Challenge, and the international semantic service selection contest (S3) [25,39].

\subsection{Centralized Search}

Semantic service matchmakers. There are quite a few tools and systems for a central directory-based search for semantic services available (cf. Table 1). The majority of currently available semantic service matchmakers exploits methods for hybrid semantic selection of semantic services in OWL-S, SAWSDL, or WSML, while only very few matchmakers are language-agnostic, or perform an adaptive selection.

For example, the semantic service matchmaker iSeM [30] performs an adaptive and hybrid semantic selection of OWL-S services. Its logic-based semantic matching of services relies on the computation of strict and approximated logical I/O concept subsumption relations, and the logical specification plugin relation. Like its predecessor OWLS-MX3 [29], it also performs non-logicbased semantic matching with different classical tokenbased text similarity measures, as well as ontologybased structural matching of signature annotation concepts. Finally, it learns how to best aggregate the results of its matching filters by use of a binary SVM relevance 


\begin{tabular}{|c|c|c|c|c|c|c|}
\hline & Services & $\mathrm{I} / \mathrm{O}$ & $\mathrm{P} / \mathrm{E}$ & logic & non-logic & adaptive \\
\hline iSeM $(2012)[30]$ & OWL-S & $\checkmark$ & $\checkmark$ & $\checkmark$ & $\checkmark$ & $\checkmark$ \\
\hline OWLS-MX3 (2012)[29] & OWL-S & $\checkmark$ & & $\checkmark$ & $\checkmark$ & $\checkmark$ \\
\hline SPARQLent (2010)[55] & OWL-S & $\checkmark$ & & $\checkmark$ & & \\
\hline $\mathrm{SeMa}^{2}(2012)[46]$ & OWL-S & $\checkmark$ & $\checkmark$ & $\checkmark$ & $\checkmark$ & \\
\hline OWLS-SLR (2010)[47] & OWL-S & $\checkmark$ & & $\checkmark$ & $\checkmark$ & \\
\hline $\mathrm{XSSD}(2013)[42]$ & OWL-S & $\checkmark$ & & $\checkmark$ & $\checkmark$ & \\
\hline OWLS-iMatcher (2008)[23] & OWL-S & $\checkmark$ & & & $\checkmark$ & \\
\hline OPOSSUM (2007)[59] & OWL-S & $\checkmark$ & $\checkmark$ & $\checkmark$ & $\checkmark$ & \\
\hline Nuwa $(2012)[25]$ & OWL-S, SAWSDL & $\checkmark$ & & $\checkmark$ & $\checkmark$ & \\
\hline SAWSDL-iMatcher (2011)[65] & SAWSDL & $\checkmark$ & & & $\checkmark$ & \\
\hline LOG4SWS (2012)[40] & SAWSDL & $\checkmark$ & & $\checkmark$ & $\checkmark$ & $\checkmark$ \\
\hline COV4SWS (2012)[40] & SAWSDL & $\checkmark$ & & & $\checkmark$ & $\checkmark$ \\
\hline SAWSDL-MX2 (2009)[33] & SAWSDL & $\checkmark$ & & $\checkmark$ & $\checkmark$ & \\
\hline URBE (2009)[54] & SAWSDL & $\checkmark$ & & & $\checkmark$ & \\
\hline TOMACO (2015)[58] & SAWSDL & $\checkmark$ & & $\checkmark$ & $\checkmark$ & \\
\hline IRS-III (2008)[11] & WSML & $\checkmark$ & & $\checkmark$ & & \\
\hline WSMO-MX (2009)[31] & WSML & $\checkmark$ & $\checkmark$ & $\checkmark$ & $\checkmark$ & \\
\hline Glue $(2005)[9]$ & WSML & $\checkmark$ & $\checkmark$ & $\checkmark$ & & \\
\hline SOA4ALL matcher (2010)[20] & WSML & $\checkmark$ & $\checkmark$ & $\checkmark$ & & \\
\hline XAM4SWS (2010)[41] & hRESTS, microWSMO & $\checkmark$ & & $\checkmark$ & $\checkmark$ & $\checkmark$ \\
\hline PSemMa (2012)[5] & prop. & $\checkmark$ & & $\checkmark$ & $\checkmark$ & \\
\hline $\operatorname{MDSM}(2008)[34]$ & PIM4SWS (meta-model) & $\checkmark$ & $(\checkmark)$ & $\checkmark$ & $\checkmark$ & \\
\hline iServe $(2010)[51]$ & MSM (meta-model) & $\checkmark$ & & $\checkmark$ & & \\
\hline
\end{tabular}

Table 1 Representative approaches for semantic service selection (matchmaking)

classifier with an evidential coherence-based weighting scheme. The logic-based semantic service matchmaker SPARQLent [55] considers the full functional profile of OWL-S services. It performs a RDF entailment rulebased matching of the OWL-S service I/O concepts, preconditions and effects described in SPARQL.

XAM4SWS [41] is a hybrid adaptive matchmaker based on LOG4SWS, which supports semantically enriched RESTful services. In particular, hRESTS and MicroWSMO are supported by means of a layered approach starting from parameter definitions up to the service level on top. Parameter matching is performed in terms of logic-based filtering combined with structural comparision of semantic annotations. Offline machine learning is applied in order to learn optimal numerical similarity assessments for logic-based filter categories. Results are aggregated and propagated to the top level by means of weighted averaging. Moreover, REST-specific details like the type of HTTP request and request metadata are considered for the overall result ranking.

The MDSM [34] matchmaker employs a meta-model for semantic services named PIM4SWS (process independent model for semantic web services) and applies semantics-preserving transformations of the information model of semantic services in OWL-S, WSML and
SAWSDL to resolve queries across different formats and formal semantics. Similarly, iServe [51] employs the MSM (Minimal Service Model) [62] on a meta level, which aims to unify both SOAP-based and RESTful web service descriptions by providing RDFS vocabulary for the largest common denominator between these formalisms. That way, it is able to match OWL-S, WSML, SAWSDL, WSMO-Lite as well as MicroWSMO and SAREST services.

Performance of matchmakers. According to the results of the international semantic service selection contest (S3) in 2012 [25], the currently best performing tools for OWL-S service selection with respect to AP are iSeM (.922), $\mathrm{SeMa}^{2}(.877)$ and Nuwa (.853). Notably, all of them are hybrid semantic matchmakers which combine logic-based I/O matching with different kinds of non-logic-based I/O similarity measurement. The offline learning of an optimal weighted aggregation of different matching filter results was essential for the highest precision achieved by the few adaptive matchmakers like iSeM. One downside of the "heavy-weight" approaches of logic IOPE-based service selection is their relatively high AQRT which ranges from $2.332 \mathrm{sec}$ (iSeM) up to $18.334 \mathrm{sec}$ (Nuwa). On the other hand, efficient indexing and preprocessing enabled the XSSD [42] match- 
maker to react very fast to queries $(\mathrm{AQRT}=.125 \mathrm{sec})$ while still being reasonably precise $(\mathrm{AP}=.795)$. For SAWSDL service selection, the tools iSeM, LOG4SWS and COV4SWS were ranked at the top with AP of .842, .837 and .823 respectively. In principle, the same conclusions can be drawn as above for the OWL-S track: Hybrid matchmaking and adaptation features pay off with respect to higher precision, but in most cases at the cost of response time. Recently, the hybrid semantic but non-adaptive SAWSDL matchmaker Tomaco [58] was reported to achieve an even higher AP of .852 with a more fine-grained logic-based and string matching filter combination than iSeM.

Public directories and search engines. At present, there are no central and authoritative directories of semantic services available in the public web. Public collections of semantic services are, for example, the prominent OWLS-TC ${ }^{4}$ for OWL-S services, the SAWSDL$\mathrm{TC}^{5}$ for SAWSDL services, and hRESTS-TC ${ }^{6}$ for annotated REST services. The service discovery platform iServe [51] can be used to build and maintain directories of semantic services in SAWSDL, OWL-S, MicroWSMO, and WSMO-Lite. The service semantics are internally represented in a minimal service model and then exposed in HTML and RDF as linked services with a unique and resolvable HTTP URI. Any iServe directory can be queried through a SPARQL endpoint. For service selection, iServe provides means of keyword search, functional classification, and service I/O parameter matching based on RDFS reasoning. Specialized search engines for semantic services are S3E [15] and Sousuo [35]. The latter performs a meta-search by utilizing the search engines Google, A9 and its own focused topic crawler, an inverse ontology-based search with the semantic web search engine Swoogle, and a full text search of the public scientific web archive citeseer. Service selection relies on full-text or keyword search in its XML-encoded service index. Alternatively, the S3E engine is encoding the profiles of crawled semantic services in RDF and selects relevant services from an internal RDF store with SPARQL.

\footnotetext{
4 http://projects.semwebcentral.org/projects/owls-tc/

5 http://projects.semwebcentral.org/projects/sawsdl-tc

6 http://projects.semwebcentral.org/projects/hrests-tc/
}

\subsection{Decentralized Search}

There are quite a few approaches for a decentralized search for semantic services in structured, unstructured or hybrid P2P networks (cf. Table 2). Most of these support the prominent semantic web service models OWLS, WSML and SAWSDL.

Search in structured P2P networks. The AGORA-P2P system [38] performs a decentralized and directory-based search for OWL-S services in a Chord ring which is used for the distributed storage and location of all services in this structured P2P network. In particular, the OWL$\mathrm{S}$ service signature concept labels are hashed as literals to unique integer keys such that peers holding the same key are offering services with equal literals in the circular key space. Service selection for multi-key queries relies on exact key matching.

Search in unstructured P2P networks. The RS2D system [2] performs a decentralized and directory-less discovery of semantic services in unstructured P2P networks. In particular, each peer dynamically builds and maintains its local view of the semantic overlay of the network, and uses the OWLS-MX matchmaker for hybrid semantic service selection. A peer also learns the average query-answering behavior of its direct neighbors in the network. The peer's decision to whom to forward a semantic service request is then driven by its estimated probabilistic risk of routing failure in terms of semantic loss and communication costs. Alternatively, peers in P2P-SDSD [3] construct a semantic overlay based on the notion of semantic links which are established between peers that provide similar services. To maintain the semantic overlay, specific protocols for joining and leaving the network as well as modifications at peers have to be executed. Moreover, peers perform random probing to counteract network dynamics and evolve the semantic overlay accordingly. Queries are forwarded along those semantic links, which match best with a given request.

Search in hybrid P2P networks. In [10], peers of a hybrid P2P system may search for relevant OWL-S, WSML and SAWSDL services based on $\mathrm{JXTA}^{7}$. Like in [61], super-peers are used to group peers based on their semantic domain expertise. Semantic service queries are

\footnotetext{
7 https://jxta.kenai.com/
} 


\begin{tabular}{|c|c|c|c|c|c|c|c|}
\hline & Services & Structured P2P & Unstruct. P2P & $\mathrm{I} / \mathrm{O}$ & $\mathrm{P} / \mathrm{E}$ & QoS & Selection \\
\hline AGORA-P2P (2006)[38] & OWL-S & Chord & & $\checkmark$ & & & Hybrid \\
\hline $\operatorname{RS} 2 \mathrm{D}(2006)[2]$ & OWL-S & & $\checkmark$ & $\checkmark$ & & & Hybrid \\
\hline Skoutas+ (2008) [57] & OWL-S & SpatialP2P $[21]$ & & $\checkmark$ & & & Non-logic \\
\hline Chord4S (2013)[18] & OWL-S & Chord & & $\checkmark$ & & & Non-logic \\
\hline Zhang+ (2009)[68] & OWL-S & & $\checkmark$ & $\checkmark$ & & $\checkmark$ & Hybrid \\
\hline Di Modica+ (2011)[10] & OWL-S, WSML, SAWSDL & & $\checkmark$ & $\checkmark$ & & & Hybrid \\
\hline ERGOT (2012) [53] & SAWSDL & Chord & & $\checkmark$ & & & Hybrid \\
\hline $\mathrm{Vu}+(2006)[63]$ & WSML & Chord & & $\checkmark$ & $\checkmark$ & $\checkmark$ & Logic \\
\hline METEOR-S (2005)[61] & WSDL-S & super-peers & random walk & $\checkmark$ & & & Non-logic \\
\hline PYRAMID-S (2009)[52] & PS-WSDL & super-peers & random walk & $\checkmark$ & & $\checkmark$ & Hybrid \\
\hline P2P-SDSD (2008)[3] & prop. & & $\checkmark$ & $\checkmark$ & & & Hybrid \\
\hline
\end{tabular}

Table 2 Representative approaches for semantic service search in P2P networks.

routed to most relevant super-peers in which groups then informed random walks are performed by the peers. For semantic relevance computation, different similarity functions can be employed by the peers in a pluginlike fashion in order to support different semantic service descriptions. Similarly, PYRAMID-S [52] performs a hybrid $\mathrm{P} 2 \mathrm{P}$ search for annotated WSDL services in a proprietary service model PS-WSDL. The P2P network is structured into synchronizing super-peers each of which with particular domain expertise and an associated group of peers. Service requests are routed to the most relevant domain super-peers in whose groups services are searched per random walk and based on non-logic-based service IO and QoS parameter matching.

Many systems for semantic data retrieval in $\mathrm{P} 2 \mathrm{P}$ networks are available, such as INGA [43], Bibster [17], GridVine [1] or S2P2P [4]. Though most of them are tailored to a specific domain such as bibliographic references in Bibster, after some reasonable modification efforts they could be used for semantic service search as well. Finally, the work on centralized and QoS-based service selection does complement QoS-related numeric constraint problem solving with reasoning on the functional semantics of services.

\section{Applications}

Semantic service selection is a key technology for building service-based applications of the converging Internet of Services, Internet of Things, and Internet of Interaction [19]. Prominent application domains include semantic business process management, smart health services, cloud manufacturing and manufacturing 4.0, and semantic social multimedia.
For example, Loskyll et al. [44] propose a system in the context of manufacturing 4.0, which makes use of semantic services in OWL-S in order to describe the functionality of manufacturing devices including sensors. The SmartFactory-KL production line is introduced as a practical use case in which both semantic service search and composition can be performed to accomplish service-based manufacturing process tasks. For example, logic-based semantic service selection with iSeM is used to search for relevant sensors which functionality is described with semantic services stored in a central sensor service directory. The related concept of semantic sensor service networks based on the utilization of the W3C semantic sensor network ontology and the adoption of semantic technologies for applications in the Internet of Things, in particular the Semantic Sensor Web, is further discussed in, for example, [64,6, 7].

In [8], semantic service selection is applied to cloud computing. In particular, cloud infrastructure (IaaS) providers describe their services in terms of semantic descriptions in WSML, which are stored in a service registry. A logic-based semantic matchmaker is deployed to enable users to select appropriate resources for their applications to be deployed in the cloud. The system is a prototype implementation. A show case of an audio/video devices online store seeking to lease new cloud resources is provided to show the feasibility of the approach.

The SADI (Semantic Automated Discovery and Integration) design pattern presented in [67] is a lightweight approach to support discovery and consumption of semantic web services based on existing standards and technologies. The specification of this pattern does not dictate a distinct service description format and provides an example application of the RDF- 
based SADI pattern and the myGrid/Moby service ontology. Service parameters are annotated in OWL-DL, and the semantic service discovery is logic-based IOPE. SADI itself does not specify a directory, but assumes service URIs as valid reference points such that a directory could be applied on top of it. SADI has been employed in two well-known semantic knowledge management tools in the domain of bioinformatics, namely the IO Informatics Knowledge Explorer, and Taverna [66]. Moreover, a prototype semantic query system called SHARE (Semantic Health And Research Environment) [60] has been implemented based on SADI and the Pellet OWL-DL reasoner.

MyMedia [32] is a mobile applications for Android, which enables users to search, share and experience videos and live recordings in a $\mathrm{P} 2 \mathrm{P}$ network of mobile devices (smartphones, tablets). For this, it combines high-precision semantic $\mathrm{P} 2 \mathrm{P}$ service search and network-adaptive P2P live streaming using the ISO/IEC standard MPEG-DASH. In particular, the semantic P2P service search for relevant video services to a given topic is implemented by combining the flexible $\mathrm{P} 2 \mathrm{P}$ semantic data discovery system S2P2P [4] with the semantic service matchmaker iSeM for local item selection. MyMedia has been prototypically implemented. Moreover, its core technologies have been applied in a real-world application, which enabled users to experience trailer videos and live streams in context of an international film festival.

\section{Conclusions}

In this paper, we provided a brief survey of approaches for centralized and decentralized search of semantic web services. There are quite a few and sophisticated techniques for this purpose with various applications in different domains which make use of them in order to achieve a more precise service selection. However, while the field of centralized semantic service search appears to be mature in this respect, that still does not hold for the decentralized case. Research challenges are concerned with, for example, the decentralized, adaptive discovery and composition of semantic services on networked resource-constrained devices in stochastic environments, the efficient interleaving of SLA negotiation with semantic composition of services for industrial service marketplaces, and a near real-time multi-objective optimisation of service-based processes at runtime with integrated re-planning of semantic services for cloud manufacturing scenarios. Besides, the trade-offs between the advantage of applying these techniques and the required computational and manual efforts compared to those needed for non-semantic service search remain to be more deeply investigated.

\section{References}

1. Aberer, K.; Cudré-Mauroux, P.; Hauswirth, M.; Van Pelt, T. (2004): Gridvine: Building internet-scale semantic overlay networks. Proc. 3rd Intern. Semantic Web Conference; Springer.

2. Basters, U.; Klusch, M. (2006): RS2D: Fast Adaptive Search for Semantic Web Services in Unstructured P2P Networks. Proc. 5th Intern. Semantic Web Conference; LNCS; Springer.

3. Bianchini, D.; De Antonellis, V.; Melchiori, M.; Salvi, D. (2008): A semantic overlay for service discovery across web information systems. Proc. Intern. Conference on Web Information Systems Engineering; Springer.

4. Cao, X.; Klusch, M. (2013): S2P2P: Semantic Search in Unstructured Peer-to-Peer Networks. Proc. 15th IEEE International Conference on High-Performance Computing and Communications (HPCC); IEEE.

5. Cassar, G.; Barnaghi, P.; Wang, W.; Moessner, K. (2012): A hybrid semantic matchmaker for IoT services. Proc. IEEE Intern. Conference on Green Computing and Communications; IEEE.

6. Compton, M.; Henson, C.; Lefort, L.; Neuhaus, H.; Sheth, A. (2009): A Survey of the Semantic Specification of Sensors. Proc. Intern. Workshop on Semantic Sensor Networks.

7. Corcho, O.; Garcia-Castro, R. (2010): Five challenges for the Semantic Sensor Web. Semantic Web Journal, 1(1-2).

8. Dastjerdi, A. V.; Tabatabaei, S. G. H.; Buyya, R. (2010): An effective architecture for automated appliance management system applying ontology-based cloud discovery. Proc. 10th IEEE/ACM Intern. Conference on Cluster, Cloud and Grid Computing; IEEE.

9. Della Valle, E.; Cerizza, D.; Celino, I. (2005): The mediator centric approach to Automatic Web Service Discovery of Glue. Proc. 1st Intern. Workshop on Mediation in Semantic Web Services.

10. Di Modica; G., Tomarchio, O.; Vita, L. (2011): Resource and service discovery in soas: a p2p oriented semantic approach. Applied Mathematics and Computer Science, 21(2).

11. Domingue, J.; Cabral, L.; Galizia, S.; Tanasescu, V.; Gugliotta, A.; Norton, B.; Pedrinaci, C. (2008): IRS-III: A broker-based approach to semantic Web services. Web Semantics, 6(2).

12. Farrell, J.; Lausen, H. (2007): Semantic Annotations for WSDL and XML Schema. www.w3.org/TR/sawsdl/

13. Fensel, D.; Lausen, H.; Polleres, A. (2010): Enabling Semantic Web Services. Springer.

14. García, J. M.; Ruiz, D.; Ruiz-Cortés, A. (2012): Improving semantic web services discovery using SPARQL-based repository filtering. Web Semantics, 17. 
15. Giantsiou, L.; Loutas, N.; Peristeras, V.; Tarabanis, K. (2009): Semantic service search engine (S3E): An Approach for Finding Services on the Web. Proc. 2nd World Summit on the Knowledge Society; Springer

16. Gomadam, K.; Ranabahu, A.; Sheth, A. (2010): SA-REST: Semantic Annotation of Web Resources. www.w3.org/Submission/2010/SUBM-SA-REST20100405/

17. Haase, P.; Schnizler, B.; Broekstra, J.; Ehrig, M.; van Harmelen, F.; Menken, M.; Mika, P.; Plechawski, M.; Pyszlak, P.; Siebes, R.; Staab, S.; Tempich, C. (2004): Bibster a semantics-based bibliographic peer-to-peer system. Proc. 3rd Intern. Semantic Web Conference; Springer.

18. He, Q.; Yan, J.; Yang, Y.; Kowalczyk, R.; Jin, H. (2013): A decentralized service discovery approach on peer-to-peer networks. Transactions on Services Computing, 6(1).

19. Issarny, V.; Georgantas, N.; Hachem, S.; Zarras, A.; Vassiliadist, P.: Autili, M. A.; Hamida, A. B. (2011): Serviceoriented middleware for the future internet: state of the art and research directions. Internet Services and Applications, $2(1)$

20. Junghans, M.; Agarwal, S.; Studer, R. (2010): Towards practical semantic web service discovery. Proc. 9th Intern. Semantic Web Conference; Springer.

21. Kantere, V.; Skiadopoulos, S.; Sellis, T. (2009): Storing and indexing spatial data in $\mathrm{p} 2 \mathrm{p}$ systems. IEEE Transactions on Knowledge and Data Engineering, 21(2).

22. Kapahnke, P.; Liedtke, P.; Nesbigall, S.; Warwas, S.; Klusch, M. (2010): ISReal: An Open Platform for SemanticBased 3D Simulations in the 3D Internet. Proc. 9th Intern. Semantic Web Conference; LNCS 6414; Springer.

23. Kiefer, C.; Bernstein, A. (2008): The Creation and Evaluation of iSPARQL Strategies for Matchmaking. Proc. 5th European Semantic Web Conference; LNCS; Springer.

24. Klusch, M. (2014): Service Discovery. In: Alhajj, R.; Rokne, J. (eds.), Encyclopedia of Social Networks and Mining (ESNAM), Springer.

25. Klusch, M. (2012): The S3 Contest: Performance Evaluation of Semantic Service Matchmakers. In: Blake, M.B.; Cabral, L.; Koenig-Ries, B.; Kuester, U.; Martin, D. (Eds.): Semantic Web Services: Advancement through Evaluation; Springer

26. Klusch, M. (2008): Semantic Web Service Description. In: [56], Chapter 3.

27. Klusch, M. (2008): Semantic Web Service Coordination. In: [56], Chapter 4.

28. Klusch, M.; Fries, B.; Sycara, K. (2006): Automated Semantic Web Service Discovery with OWLS-MX. Proc. 5th Intern. Conference on Autonomous Agents and MultiAgent Systems; ACM.

29. Klusch, M.; Kapahnke, P. (2009): OWLS-MX3: An Adaptive Hybrid Semantic Service Matchmaker for OWL-S. Proc. 3rd Intern. Workshop on Semantic Matchmaking and Resource Retrieval (SMR2); CEUR.

30. Klusch, M.; Kapahnke, P. (2012): The iSeM Matchmaker: A Flexible Approach For Adaptive Hybrid Semantic Service Selection. Web Semantics, 15.

31. Klusch, M; Kaufer, F. (2009): WSMO-MX: A Hybrid Semantic Web Service Matchmaker. Web Intelligence and Agent Systems, 7(2).
32. Klusch, M.; Kapahnke, P.; Cao, X.; Rainer, B.; Timmerer, C.; Mangold, S. (2014): MyMedia: Mobile Semantic Peer-to-Peer Video Search and Live Streaming. Proc. 11th ACM Intern. Conference on Mobile and Ubiquitous Systems; ACM.

33. Klusch, M.; Kapahnke, P.; Zinnikus, I. (2011): Adaptive Hybrid Semantic Selection of SAWSDL Services with SAWSDL-MX2. Semantic Web and Information Systems, $6(4)$.

34. Klusch, M.; Nesbigall, S.; Zinnikus, I. (2008): ModelDriven Semantic Web Service Matchmaking for Collaborative Business Processes. Proc. 2nd Intern. Workshop on Semantic Matchmaking and Resource Retrieval, CEUR 416.

35. Klusch, M.; Xing, Z. (2008): Deployed Semantic Services for the Common User of the Web: A Reality Check. Proc. 2nd IEEE Intern. Conference on Semantic Computing; IEEE.

36. Kona, S.; Bansal, A.; Simon, L.; Mallya, A.; Gupta, G.; Hite, T.D. (2009): USDL: A Service-Semantics Description Language for Automatic Service Discovery and Composition. Web Services Research.

37. Kopecky, J.; Gomadam, K.; Vitvar, T. (2008): hRESTS: An HTML Microformat for Describing RESTful Web Services. Proc. IEEE Intern. Conference on Web Intelligence and Intelligent Agent Technology.

38. Küngas, P.; Matskin, M. (2006): Semantic web service composition through a p2p-based multi-agent environment. Proc. Intern. Workshop on Agents and Peer-to-Peer Computing, Springer.

39. Kuester, U.; Koenig-Ries, B.; Klusch, M. (2009): Evaluating Semantic Web Service Technologies: Criteria, Approaches and Challenges. In: Progressive Concepts for Semantic Web Evolution: Applications and Developments; Advances in Semantic Web Information Systems series. IGI Global.

40. Lampe, U.; Schulte, S; (2012): Self-Adaptive Semantic Matchmaking Using COV4SWS.KOM and LOG4SWS.KOM. In: B. Blake et al. (eds.) Semantic Web Services, Chapter 9, Springer.

41. Lampe, U.; Schulte, S.; Siebenhaar, M.; Schuller, D.; Steinmetz, R. (2010): Adaptive matchmaking for RESTful services based on hRESTS and MicroWSMO. Proc. 5th Intern. Workshop on Enhanced Web Service Technologies, ACM.

42. Li, J. (2013): A Fast Semantic Web Services Matchmaker for OWL-S Services. Networks, 8(5), 1104-1111.

43. Loser, A.; Staab, S.; Tempich, C. (2007): Semantic social overlay networks. Selected Areas in Communications, 25(1).

44. Loskyll, M.; Schlick, J.; Hodek, S.; Ollinger, L.; Gerber, T.; Pirvu, B. (2011): Semantic service discovery and orchestration for manufacturing processes. Proc. 14th IEEE Intern. Conference on Emerging Technologies and Factory Automation, IEEE.

45. Martin, D.; Burstein, M.; Hobbs, J.; Lassila, O.; McDermott, D.; McIlraith, S.; Narayanan, S.; Paolucci, M.; Parsia B.; Payne, T.; Sirin, E.; Srinivasan, N.; Sycara, K. (2004): OWL-S: Semantic Markup for Web Services. www.w3.org/Submission/OWL-S/

46. Masuch, N.; Hirsch, B.; Burkhardt, M.; Heler, A.; Albayrak, S. (2012): SeMa2: A Hybrid Semantic Service 
Matching Approach. In: B. Blake et al. (eds.) Semantic Web Services, Chapter 3, Springer.

47. Meditskos, G.; Bassiliades, N. (2010); Structural and role-oriented web service discovery with taxonomies in OWL-S. IEEE Transactions on Knowledge and Data Engineering, $22(2)$.

48. Oberle, D.; Barros, A.; Kylau, U.; Heinzl, S. (2013): A unified description language for human to automated services. Information Systems, 38(1).

49. Pedrinaci, C.; Leidig, T. (2011): Linked USDL Core. www.linked-usdl.org/ns/usdl-core

50. Pedrinaci, C.; Domingue, J.; Sheth, A. (2011): Semantic web services. In: Handbook of semantic web technologies. Springer.

51. Pedrinaci, C.; Liu, D.; Maleshkova, M.; Lambert, D.; Kopecky, J.; Domingue, J. (2010): iServe: a linked services publishing platform. CEUR 596.

52. Pilioura, T.; Tsalgatidou, A. (2009): Unified publication and discovery of semantic web services. ACM Transactions on the Web, 3(3).

53. Pirró, G.; Talia, D.; Trunfio, P. (2012): A dht-based semantic overlay network for service discovery. Future Generation Computer Systems, 28(4).

54. Plebani, P.; Pernici, B. (2009): URBE: web service retrieval based on similarity evaluation. IEEE Transactions on Knowledge and Data Engineering, 21(11). , IEEE.

55. Sbodio, M.L.; Martin, D.; Moulin, C. (2010): Discovering Semantic Web services using SPARQL and intelligent agents. Web Semantics, 8(4).

56. Schumacher, M.; Helin, H.; Schuldt, H. (Eds.) 2007: CASCOM - Intelligent Service Coordination in the Semantic Web. Springer.

57. Skoutas, D.; Sacharidis, D.; Kantere, V.; Sellis, T. (2008): Efficient semantic web service discovery in centralized and p2p environments. Proc. 7th Intern. Semantic Web Conference, LNCS, Springer.

58. Stavropoulos, T. G.; Andreadis, S.; Bassiliades, N.; Vrakas, D.; Vlahavas, I. (2015): The Tomaco Hybrid Matching Framework for SAWSDL Semantic Web Services IEEE Transactions on Services Computing, 99.

59. Toch, E.; Gal, A.; Reinhartz-Berger, I.; Dori, D. (2007): A semantic approach to approximate service retrieval. $A C M$ Transactions on Internet Technology, 8(1).

60. Vandervalk, B. P.; McCarthy, E. L.; Wilkinson, M. D. (2009): SHARE: A semantic web query engine for bioinformatics. Proc. Intern. Semantic Web Conference; LNCS; Springer.

61. Verma, K.; Sivashanmugam, K.; Sheth, A.; Patil, A.;. Oundhakar, S.; Miller, J. (2005): METEOR-S WSDI: A Scalable P2P Infrastructure of Registries for Semantic Publication and Discovery of Web Services. Information Technology and Management, 6(1).

62. Vitvar, T.; Kopecky, J.; Viskova, J.; Fensel, D. (2008): WSMO-Lite annotations for web services. Proc. 5th European Semantic Web Conference (ESWC); Springer.

63. Vu, L.-H.; Hauswirth, M.; Porto, F.; Aberer, K. (2006): A search engine for QoS-enabled discovery of semantic web services. Business Process Integration and Management, 1(4), 244-255.

64. Wang, W.; Barnaghi, P.; Cassar, G.; Ganz, F.; Navaratnam, P. (2012): Semantic Sensor Service Networks. Proc. IEEE Intern. Conference on Sensors; IEEE.
65. Wei, D.; Wang, T.; Wang, J.; Bernstein, A. (2011): SAWSDL-iMatcher: A customizable and effective Semantic Web Service matchmaker. Web Semantics, 9(4).

66. Withers, D.; Kawas, E.; McCarthy, L.; Vandervalk, B.; Wilkinson, M. (2010): Semantically-guided workflow construction in Taverna: the SADI and BioMoby plug-ins. In: T. Margaria et al. (eds.); Leveraging Applications of Formal Methods, Verification, and Validation; pp. 301-312; Springer.

67. Wilkinson, M. D.; Vandervalk, B. P.; McCarthy, E. L. (2011): The Semantic Automated Discovery and Integration (SADI) Web service Design-Pattern, API and Reference Implementation. Biomedical Semantics, 2(8).

68. Zhang, Y.; Huang, H.; Yang, D.; Zhang, H.; Chao, H.C.; Huang, Y.-M. (2009): Bring QoS to p2p-based semantic service discovery for the universal network. Personal and Ubiquitous Computing, 13(7). 